\title{
Numerical Modelling and Flow Analysis of a Centrifugal Pump running as a Turbine: Unsteady Flow Structures and Its Effects on the Global Performance
}

\author{
Santolaria Morros, Carlos; Fernández Oro, Jesús Manuel; \\ Argüelles Díaz, Katia María.
}

Universidad de Oviedo, Área de Mecánica de Fluidos.

Campus de Viesques, 33271, Gijón (Asturias), Spain.

jesusfo@uniovi.es

\begin{abstract}
The capability of centrifugal pumps to operate as centripetal turbines was established by several authors many years ago (Kittredge, Knapp, Stepanoff) [1-3]. More recently, Shafer, Engeda and Priesnitz [4-6] have demonstrated how the efficiency as a pump can be maintained when the machine runs as a turbine. Also, they offered relations between the flow rate and head for optimum conditions in both modes. These works concern the overall performances of these machines, based on experimental methodologies.

The purpose of this paper is to investigate the flow pattern in a centrifugal pump when it works as a centripetal turbine, with special interest in the unsteady behaviour, in order to explain the shape of the performance curves. Also, we focus on the determination of the radial thrust and other mechanical loads over a pump-design machine. The pump studied is commercial, with single axial suction and a vaneless spiral volute casing.

A numerical study has been carried out in order to obtain more information about the flow into the volute and the impeller. A numerical 3D unsteady simulation has been developed using a commercial code that solves the URANS set of equations with a standard $k$ - $\varepsilon$ turbulence model. The results show the non-axisymmetric flow developed in the volute, which implies a significant radial thrust, the interaction between the tongue and the impeller generates force fluctuations, the velocity and pressure distributions inside the impeller, and the exit flow, with post-rotation and lowpressure. These flow results allow us to understand the behaviour of the machine, by comparing it with the pump mode.

Complementarily, an experimental study has been developed in a hydraulic set-up designed specifically according to standards. It uses an auxiliary pump that supplies the flow rate and head required for the pump to work as a turbine, which is further coupled to an electric generator. This test facility allows us to characterize the pump-turbine performances in order to compare the characteristic curves in both modes and determine the performance curves at constant head. Furthermore, pressure taps located around the impeller at the front side of the volute allow the measurement of stationary and non-stationary circumference pressure distributions in order to calculate the steady radial thrust and its fluctuations. Finally, a three-hole probe, placed at the suction outlet, is used to measure the radial distribution of the velocity circumference component, which enables us to explain the loss of efficiency when postrotation appears, as well as the susceptibility to cavitation.
\end{abstract}

\section{INTRODUCTION}

The present investigation analyzes the inverse flow established in a centrifugal pump when it is operated as a turbine. None of the classical flow control devices employed in the case of radial machines designed as turbines are available for this particular application. As a consequence, the flow entering in the runner is not conditioned by a distributor, so the admission of the turbine cannot be regulated. Furthermore, since the geometry of the pump operated as a turbine is completely fixed, it is expected that considerable deviations of the primary flow may lead to a notable decrease in the performance and efficiency of the machine. On one hand, the flow patterns developed in the volute will present different features to those established when the machine operates as a pump. Thus, the inflow streamlines cannot be guided towards the axial direction every time the mass flow rate is modified, so the best efficiency point must obviously be 
far beyond the design conditions of the machine, with profile losses increased at the inlet. On the other hand, this fact is related to a poorer guidance of the flow within the runner passages, leading to excessive postrotation at the runner outlet and higher levels of residual dynamic pressure (linked to the tangential component). This component is dissipated at the machine discharge, thus increasing the mixing losses and reducing the actual work available for power transfer to the shaft.

The use of commercial pumps as turbines for smallscale hydroelectric plants is an interesting strategy for renewable power generation, due to their reduced costs associated [7]. In the literature, several authors have demonstrated that the used of pumps instead of turbines deserves consideration because, though there is an efficiency reduction, the global performance makes them really competitive [8-10]. Traditionally, these studies were focused on the experimental analysis of figures of merit, assuming similarity between maximum efficiencies working in both modes, and proposing mathematical fittings to advance the inverse characteristics [11-12]. Nowadays, the introduction of numerical methodologies to analyze internal flows offers new challenges and possibilities to characterize the performance of pumps running as turbines [13-14].

In this work, a numerical simulation of a pumpdesigned radial machine operating as a turbine is developed, and validated through corresponding experimental data. The study is focused on the analysis of the basic characteristics of the flow, providing insight on the unsteady features of the energy transfer. Although the impeller blades are equally spaced, a nonaxisymmetric distortion is introduced on the flow entering the impeller, due to the peripheral restriction caused by the volute tongue. Therefore, significant unsteady fluctuations over the blade loadings can be developed, inducing important mechanical forces and imbalanced axial thrusts over the machine bearings. This effect could have a notable impact on the time life of the machine operating as a turbine.

The final purpose of the paper is the evaluation of the performance of small to medium centrifugal pumps when are operated as turbines. It will be demonstrated that they can provide acceptable efficiencies, obviously lower than in the design configuration, but high enough to offset the expensive manufacturing costs of specifically-designed hydraulic turbines with respect to the reduced prices of commercial pumps, as a possible application to small hydraulic resources.

HYDRAULIC FACILITY AND EXPERIMENTAL TESTS
A hydraulic facility has been employed to characterize the overall behaviour of a commercial pump working as a turbine. It has been designed according to International Standards, ISO 3555:1977. A second driver pump is used to force the commercial pump under research to work as a turbine. The head and flow rate required for the experimental data set are fixed by the driver pump, reaching maximum values of $90 \mathrm{~m}$ and $180 \mathrm{~m}^{3} / \mathrm{h}$, respectively. This pump aspirates from an enclosed $100 \mathrm{~m}^{3}$ reservoir and obliges the flow to enter into the spiral volute of the pump under research, transferring part of its energy to the machine and returning to the tank through the suction pipe.

The radial flow pump to be tested as a turbine has a single axial discharge tube and a vaneless volute casing. It is a seven backward curved blade commercial pump, with the most important characteristics summarized in table 1 .

\begin{tabular}{lc}
\hline Impeller outlet diameter $\left(\mathrm{D}_{2}\right)$ & $200 \mathrm{~mm}$ \\
\hline Impeller outlet width $\left(\mathrm{b}_{2}\right)$ & $16.84 \mathrm{~mm}$ \\
\hline Outlet blade angle $\left(\beta_{2}\right)$ & $29^{\circ}$ \\
\hline Tip inlet diameter $\left(\mathrm{D}_{1 \mathrm{t}}\right)$ & $88 \mathrm{~mm}$ \\
\hline Hub inlet diameter $\left(\mathrm{D}_{1 \mathrm{~h}}\right)$ & $67 \mathrm{~mm}$ \\
\hline Rotacional speed $(\omega)$ & $1500 \mathrm{rpm}$ \\
\hline Blade passing frequency $(\mathrm{BPF})$ & $175 \mathrm{~Hz}$ \\
\hline Nominal flow rate $\left(\mathrm{Q}_{\mathrm{N}}\right)$ & $0.015 \mathrm{~m}^{3} / \mathrm{s}$ \\
\hline Head at nominal conditions $\left(\mathrm{H}_{\mathrm{N}}\right)$ & $12.88 \mathrm{~m}$ \\
\hline Specific speed $\left(\omega_{\mathrm{S}}\right)$ & 0.52 \\
\hline
\end{tabular}

Table 1. Main parameters of the centrifugal pump.

Different types of instrumentation have been required to characterize both global and local flow variables. In order to determine the performance curves, an orifice flowmeter, previously calibrated with a Pitot tube according to ASME indications has been mounted on the inlet duct. The flow rate is measured using a differential pressure transducer with a 0-2 bar resolution. Also, the head is measured using piezoresistive pressure transducers for a 0-20 bar range, connected to a multiplexor unit. A FAST Technology torquemeter is also mounted on the machine shaft. The torque range is $0-175 \mathrm{Nm}$ with an uncertainty less than $\pm 0.2 \%$ for all the set of experiments, so it is possible to determine the efficiency through the comparison of the mechanical power (the speed rate is obtained with an optic tachometer) with respect to the hydraulic power. 


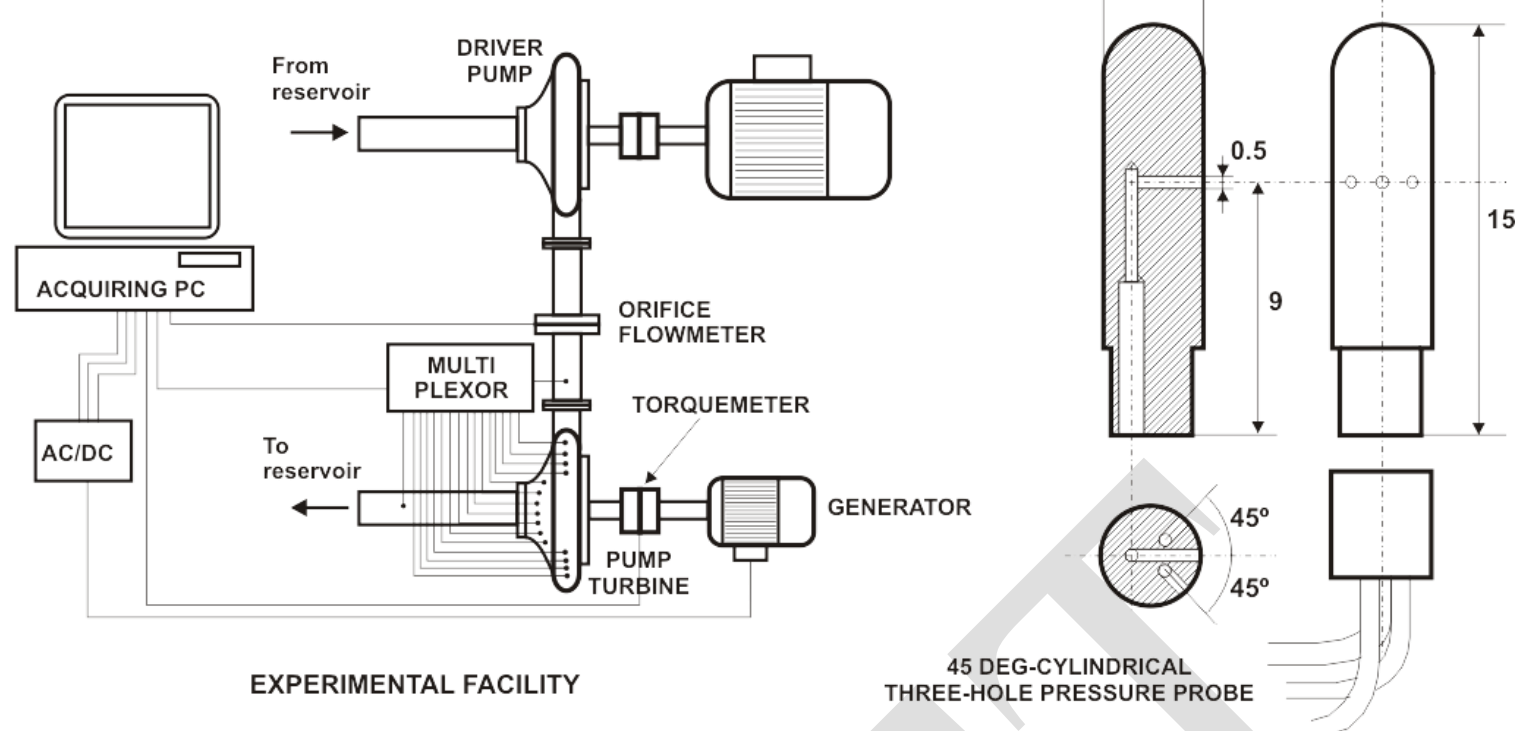

Fig. 1. Sketch of the experimental facility and the pneumatic directional pressure probe.

To determine the pressure distribution along the volute shroud, 12 pressure taps were located every 30 deg in the mid-plane circumference of the casing. The integration of the pressure signal allows us to estimate the direction and magnitude of the time-mean force over the volute as a function of the operating conditions. The signals are introduced into a computer using an $\mathrm{A} / \mathrm{D}$ data acquisition card. A data acquisition program allows the temporal averaging of the signals at each working point.

In addition, a cylindrical three-hole pressure probe has been built ad hoc to provide intrusive, local measurements of the flow patterns inside the volute and at the machine discharge. The holes, $0.5 \mathrm{~mm}$ dia., with an angular distance between them of $45 \mathrm{deg}$, provide the pneumatic pressure in the sensors that is further reduced according to the calibration curves of the probe to obtain the local static and total pressure. The angular coefficient of the calibration recovers the flow angle, whereas the inferred dynamic pressure provides the local velocity magnitude. Typical calibration coefficients have been employed, with a maximum angular range of $\pm 30 \mathrm{deg}$, meeting the angular span required. The uncertainty in the angle measurement is lower than $0.5 \mathrm{deg}$ all along the angular range of the probe, with a velocity uncertainty kept below $0.3 \%$ for maximum velocities of $20 \mathrm{~m} / \mathrm{s}$ to be measured. More details of the hydraulic facility and the pressure probe can be found in [15].

\section{NUMERICAL MODELING}

Geometry, grid and flow solver. For the present investigation, a formerly well-tested numerical model (employed for centrifugal pumps working in direct mode) has been employed [16]. The model, implemented on the commercial code FLUENT, presents a reliable spatial discretization, with additional refinement at those zones where important gradients are expected to be found: close to the tongue region and all along the runner blades and passages. A hybrid meshing strategy has been followed to provide an efficient refinement towards the endwalls, following the guidelines proposed by Freitas [17]. Structured hexahedral cells have been employed to define both inlet and outlet zones, whereas unstructured tetrahedral cells have been piled up towards the endwalls of the impeller and the volute. The grid generated is shown in figure 2. Grid interfaces are introduced to exploit the sliding mesh technique implemented by the FLUENT code. This technique allows for the relative motion of the impeller with respect to the volute casing, so a full unsteady simulation can be run. Also, the inclusion of these two interfaces (Fig. 2) has allowed the introduction of a non-conformal grid, which is simpler to implement.

The commercial CFD software FLUENT v6.3 was used to solve the Navier-Stokes set of equations. This $3 \mathrm{D}$ viscous code introduces a cell-centered volume finite method with second-order upwind schemes for convection and diffusion terms. A SIMPLEC algorithm is chosen for the pressure and velocity coupling. Turbulence is simulated with the standard $k-\varepsilon$ model, introducing wall functions based on the logarithmic law. This choice has been demonstrated to provide good results when simulating centrifugal pumps, capturing with high reliability the details of the flow near the tongue and even the jet-wake structure [18]. Finally, a second order, implicit scheme has been introduced for the time dependent term of the governing equations. Special care was taken in the election of the time step required to obtain an accurate temporal resolution of the blade passing scales. More details are discussed below in the section describing the characteristics of the unsteady solution. 


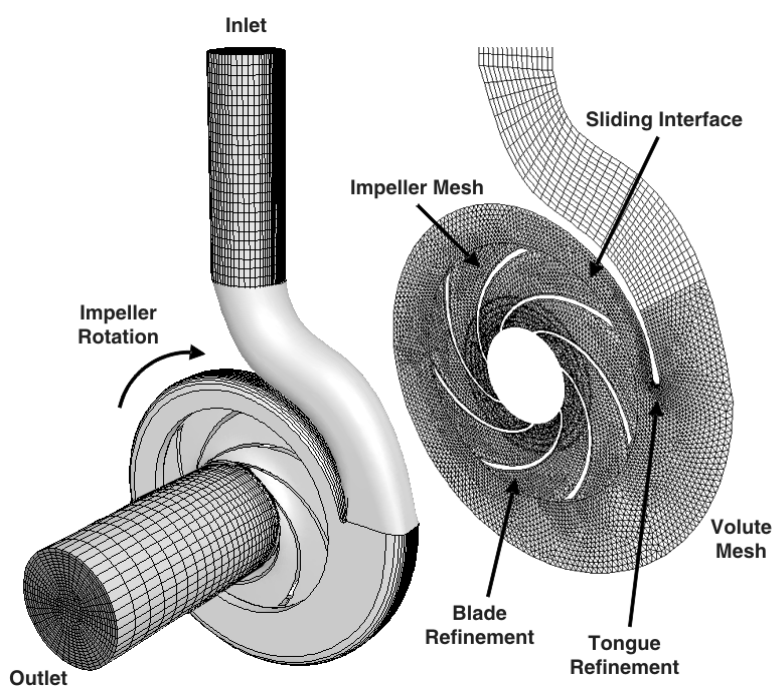

Fig. 2. Geometry and mesh of the numerical modelling.

Boundary conditions. The boundary conditions represent an important constraint for the complete definition of the numerical model. It is desirable that they stand a real physical meaning enhancing the turbomachinery flow calculations. In the present investigation, static pressure at the inlet and zero total pressure at the outlet have been chosen as the most neutral conditions. Thus, the value of the static pressure at the inlet represents the net head supplying energy to the turbine, while a zero outlet total pressure gives an idea of the atmospheric conditions at the machine discharge. The operating conditions of the machine are changed by modifying the value of the net head at the inlet, ranging from 10 to 65 meters. These boundary conditions avoid the definition of a uniform velocity profile at the inlet or outlet, generally non realistic. Notice that it is necessary to place these conditions far beyond the machine geometry in order to preserve the resolution of the internal flow from being conditioned by the constant values, typically several diameters away. In addition, the no-slip condition is imposed on all the impeller blades and sidewalls in the relative frame, as well as on the volute casing and both inlet and outlet pipe walls in the fixed frame.

Unsteady solution characteristics. The solution procedure starts with a "rotor frozen" simulation, in order to approach the initialization of all the variables to the real time-varying gradients within the impeller. Afterwards, the sliding mesh technique is applied, allowing the model to work over several turns until a periodic, statistically stable state is reached. Finally, a definitive execution during two rotor revolutions is conducted for every operating point, in advance for a forward post-processing of the flow solution, giving worthwhile average time and RMS fluctuation figures. It has been observed that the election of an accurate time step is a critical issue for the turbine operation mode. Otherwise, large variations in the torque response arise, outside the statistical controls established for an accurate periodic description of the unsteady flow. An exhaustive analysis of the time step dependence has been carried out, ranging from typical sizes (18 to 30 time steps per blade passing frequency), to extremely fine resolutions, comprising 45 to 90 time steps describing one impeller passage. This means that the performance of a complete rotor revolution is described each 630 steps. Basically, those operating points far beyond the best efficiency point (BEP) required up to 90 time steps per passage, whereas conditions close to the BEP were fully resolved using 75 or even 60 time steps. Therefore, an adaptive strategy for the time resolution was adopted; setting a minimum time step of $6.34 \times 10^{-5} \mathrm{sec}$ in the worst cases. Complementarily, the number of iterations per time step has been adjusted to reduce the residuals below a typical criterion of $10^{-6}$. Furthermore, the residuals, defined as the imbalance of the numerically-resolved equations, were monitored to check that no convergence improvement was fulfilled by increasing the number of iterations. Thus, typical values ranging from 50 to 75 iterations per time step were fixed during the computations.

The complete iterative routine was executed over a parallelized cluster (Athlon-K7, $500 \mathrm{Mhz}$ ), requiring approximately 150 hours of CPU time to achieve the fully periodic unsteady solution for every point simulated in the performance curve.

\section{OVERALL FLOW VARIABLES}

The analysis of the numerical model will provide a deep knowledge of the local characteristics of the internal flow within the impeller, especially in the volute tongue region. However, it is necessary to validate the results obtained by the code, in order to assess the reliability of the methodology. For that purpose, a global analysis of the pump operating in the inverse mode is carried out as the starting point. In particular, a comparison of the experimental data with respect to the numerical results will allow us to calibrate the soundness of the numerical scheme.

Figure 3 shows a comparison of the figures obtained for the turbomachine. As a function of the flow coefficient, defined as $\phi=Q / \omega D^{3}$, both the head coefficient, $\psi=g H / \omega^{2} D^{2}$, and the efficiency, $\eta$, are represented in the plot in black and grey respectively. Solid lines represent for the experimental data, while all the numerical points simulated have been represented with square and round dots. Although the numerical model considers neither the disk friction losses nor the mechanical losses at the bearings, there is an encouraging overall agreement between curves. The experimental best efficiency point for the pump working as a turbine is close to $\phi=0.015$, with greatest efficiencies reaching close to $80 \%$. Furthermore, the typical evolution of the performance curve of a centrifugal turbine is perfectly reproduced in the modelling. Only at higher flow rates do the discrepancies between numerical and experimental data arise. In addition, far beyond the BEP, both numerical 
and experimental results exhibit a progressive decrease in efficiency, clearly due to the absence of any control device for the turbine admission in off-design conditions. The extrapolation of the performance curves towards the vertical axis is an indicator of the energy that is dissipated by the machine due to mechanical losses. As expected, head consumption is required for zero-flow conditions. This can be also observed in the distribution of the torque, as zero-torque is not reached when the machine discharges no flow rate, but when there is still a marginal head consumption.

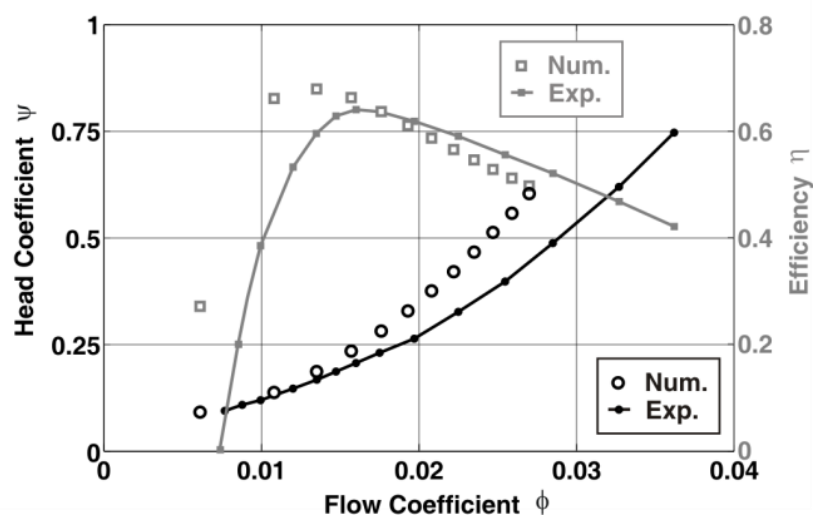

Fig 3. Comparison of the performance curves between numerical and experimental results.

A representation of both numerical torque and axial thrust is plotted in figure 4 . The computations predict a progressive increase in both magnitudes, suggesting a quadratic trend with the increasing flow rate. As the rotational speed remains constant, the raising torque is an indicator of the increasing power in the shaft. These results are completely coherent with one-dimensional flow theories, which predict a quadratic dependency of the hydraulic power with respect to the flow coefficient. Accordingly, the axial thrust is also increased, as expected from dynamic considerations derived from the axial momentum equation, applied over a generic volume control enclosing the turbine impeller:

$$
F_{a x} \approx \int_{V C} \rho Q \vec{v} \cdot d \vec{S} \propto f\left(Q^{2}\right)
$$

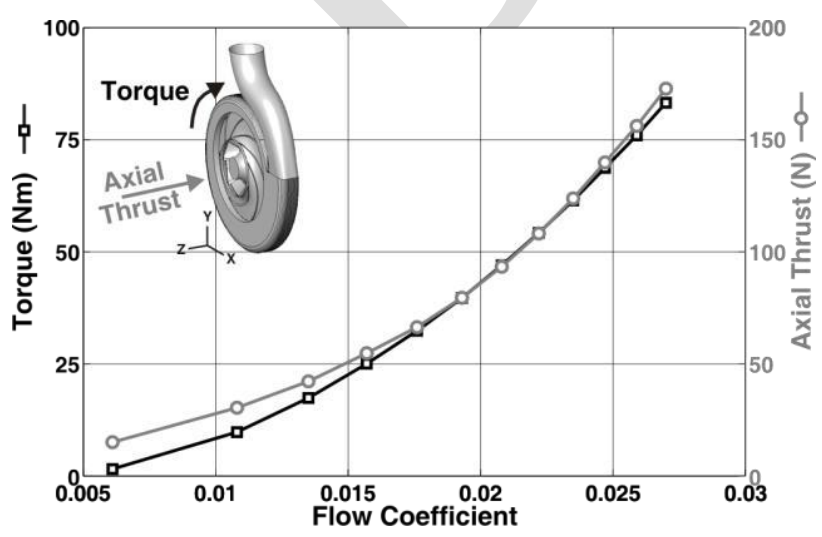

Fig 4. Numerical estimation of the torque and the axial thrust of the pump-turbine.
The radial forces exerted over the impeller are also important in order to evaluate the dynamic characteristics of the pump working as a turbine. Due to the non-axysymmetric characteristics of the inflow around the volute, the time-averaged distribution of the static pressure induces an unbalanced radial force in the impeller. This force is transmitted to the mechanical supports of the machine, conditioning its normal operation and compromising the time life of the impeller. An estimation of the radial force, especially in the analysis of the turbine mode, can be notably useful to evaluate the possibilities of the pump in the inverse mode. Figure 5 presents different results concerning the amplitude and direction of the radial forces exerted over the impeller. The forces were calculated in the absolute coordinate system where the reference for the angle is shown in the figure. The positive angular direction is the counter-rotating one (the same reference is adopted throughout the paper). In the left-hand plot, a polar representation of the mean force over the impeller, as a function of the flow coefficient, shows a progressive increase of the force with the flow rate. In contrast to the pumping mode, where a minimum value (practically negligible) is observed for the design operating point [19], the turbine suffers a radial force (approximately 30 $\mathrm{N}$ ) even for the best efficiency point. It is remarkable that the magnitudes of the radial force are similar to the values obtained for the turbomachine working as a pump [19]. Moreover, a direct comparison of similar diagrams shows that close to the design and nominal conditions, the radial forces are greater when the machine operates as a turbine, while far beyond these optimal conditions, the radial force increase is more abrupt for the pumping mode. This may be a consequence of the severe blocking effect produced by the volute tongue in the pumping mode at high flow rates. In the inverse mode, the discharge is vaneless and not restricted by the tongue, so just the profile losses affect the amplitude of the radial force.

Additionally, the reciprocating radial force acting over the volute casing may provide a complementary picture of the mechanical strains. Thus, a representation of the radial force (in both magnitude and angle values) over the volute casing is shown in the right-hand plot of the figure 5. In this case, a comparison between the numerical results (solid lines) and the experimental data is available from pressure transducers placed all around the volute in the experimental facility. To obtain the force, both experimental and numerical results have been integrated over the complete periphery of the casing, in order to compute a net, averaged radial force. The numerical model captures satisfactorily the global trends for both angles and magnitudes of the integrated force for the whole range of flow rates considered. However, the magnitude of the force is slightly overpredicted in the modelling, probably as a consequence of the moderate density of the mesh close to the sidewalls of the volute shroud. On the other hand, the distribution of the force angle reveals a clock-wise angular displacement of the radial force in a progressive, continuous fashion. The analogous result 
for the pumping mode differs substantially, since an abrupt change in the direction of the force was observed when the operating point changes from flow rates under the design conditions to flow rates beyond the nominal point [19]. In particular, positive values of the angle (near to $90 \mathrm{deg}$ ) are suddenly inverted to negative values $(-70$ to $-90 \mathrm{deg})$ when the nominal flow rate is exceeded. Once again, the restriction induced by the tongue is critical for the pumping mode, but with no significant contribution over the machine forces when working as a turbine.
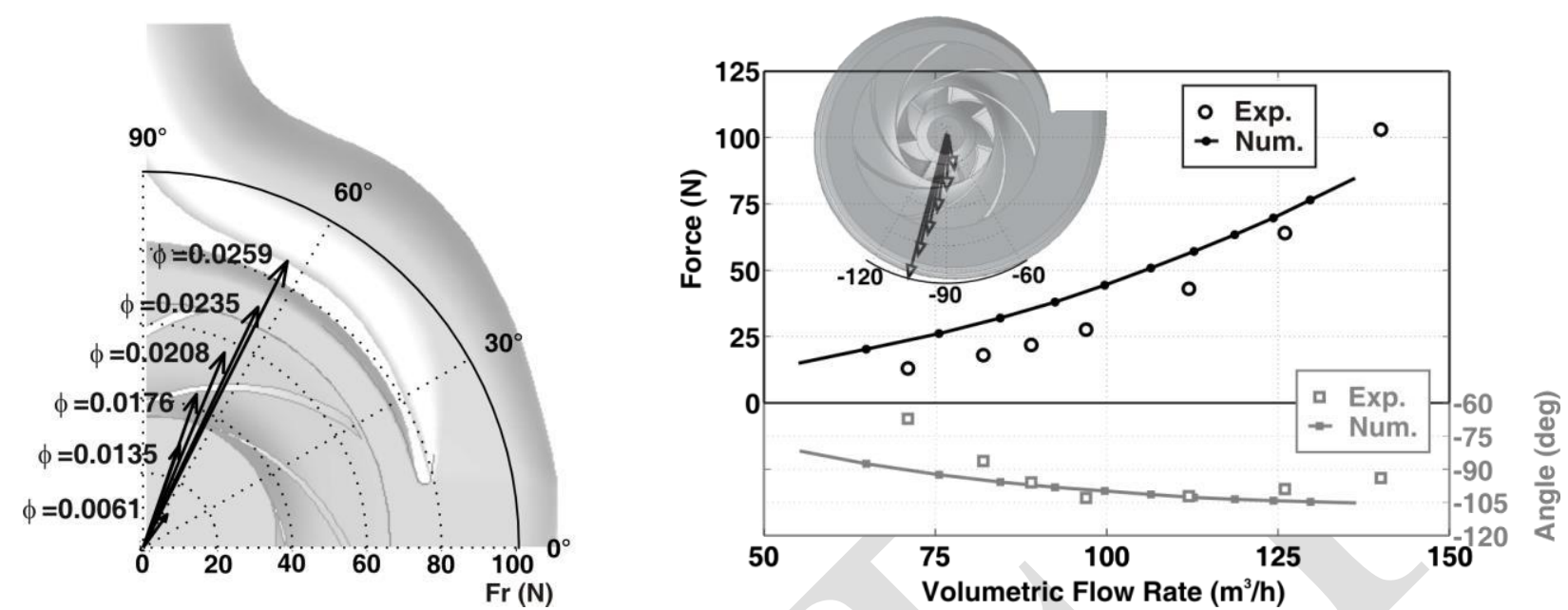

Fig. 5. Time-mean forces over the volute and the impeller as a function of the mass flow rate.

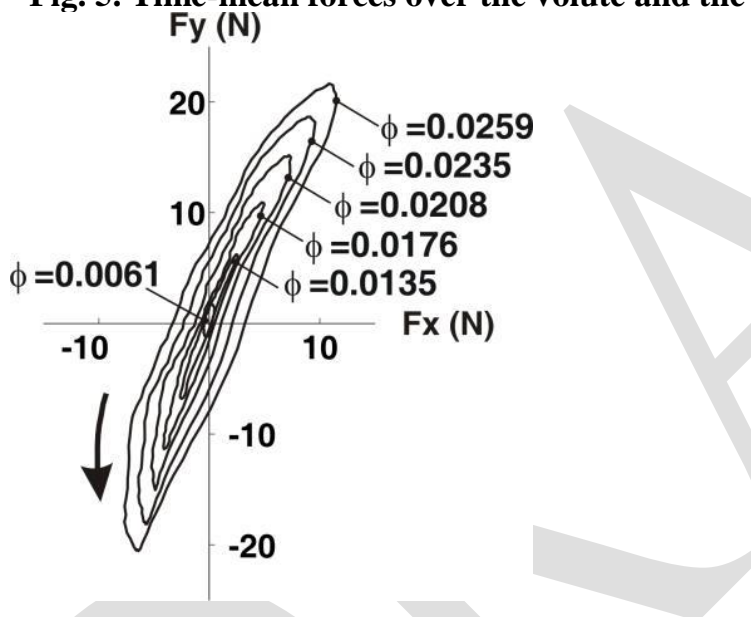

the unsteady force must present a clear periodic behaviour, developed at the blade passing frequency. Figure 6 represents the oscillation of the radial force, once the mean value, discussed in figure 5 , has been removed from the signal. The results trace an envelope for a force vector that is rotating at the blade passing frequency, that is, the force completes seven cycles for each rotation of the impeller. The force vector, placed in the origin of coordinates, evolves in an elliptical curve, rotating in the opposite direction to the impeller's rotation. Previous investigations of the authors for the pumping mode [19] revealed a similar morphology of these radial fluctuations, but rotating in the same direction as the impeller's rotation. The key point is the magnitude of the oscillations, which can be significantly large, especially for high flow rate conditions. Even for the best efficiency point, it is possible to observe an important dynamic component, with a maximum as high as $\pm 10 \mathrm{~N}$, which means $25 \%$ of the value of the steady component. A very interesting fact concerning the turbine mode is that, although the values of the mean forces are similar (even lower at high flow rate conditions) to those observed in the pumping mode, the amplitudes of the unsteady fluctuations are severely increased with respect to the design parameters of the pump as manufactured. Therefore, from the analysis of the forces, it may be concluded that this type of commercial pump can be used in an inverse mode with no excessive inconveniences: all the mechanical forces are kept within controlled limits, in the range of the specified maximum loads supported by the shaft and bearing arrangements. Also, acceptable efficiency rates (up to $75 \%$ ) are achieved, particularly in the region close to the best efficiency point. However, the time life of the machine can be seriously compromised due to the high level of unsteadiness found in the radial force. A prolonged use of the pump working as a turbine can lead to fatigue damage, needing frequent maintenance. 
Anyway, a moderate use of the pump, limited to a few hours, can satisfactorily match the requirements of small hydraulic resources, even more so if the working conditions are restricted to nominal conditions of the performance curve.

\section{FLOW FIELD ANALYSIS IN A PUMP WORKING AS A TURBINE}

Basic trends in the volute. Intrusive measurements, using the pneumatic three-hole pressure probe shown in figure 1, have been conducted circumferentially along the volute. Due to the small gap between the impeller and the volute casing, only a few representative positions were acquired along the radial coordinate. Figure 7 shows a comparison of the streamwise velocity in the volute between the experimental data and the numerical predictions. The measurements obtained with the pneumatic probe are directly compared to the timeaveraged results given by the unsteady simulation. Three different sections have been analysed to track the evolution of the flow patterns inside the volute, denoted as $\mathrm{A}, \mathrm{B}$ and $\mathrm{C}$. In section $\mathrm{C}$, the larger distance between the impeller and the sidewall has allowed measurements in three radial positions, while just two positions were accessible in sections A and B. Also, three different operating points have been compared in this analysis: the best efficiency point (dark grey) and a higher (black) and a lower (light grey) flow rate condition. The figure shows that there is reasonable agreement between the radial distributions of the numerical streamwise velocity (solid lines) and the corresponding experimental points. Major differences are observed close to the volute sidewalls (locations 2 and 3 ) due to the underprediction of the numerical boundary layers derived from coarser meshes. Anyway, overall trends are reproduced by the numerical code: the velocity magnitude increases towards the admission of the impeller (because of the convergent flow into the runner inlet), whereas the mean velocity at every section remains approximately constant for every flow condition, in accordance with one-dimensional predictions derived from the logarithmic profile of the volute geometry [18]. Thus, the flow is uniformly delivered along the periphery of the volute, leading to a balanced full admission. Other characteristics of the primary patterns of the flow are also captured by the numerical model, e.g. the partial mixing-out of the tongue wake in section $\mathrm{C}$. Since the pneumatic probe was not placed facing the tongue, a direct comparison of the velocity deficit in that position is not available.

Similar conclusions could be derived from the comparison of the flow angle, though, for the sake of brevity, they are not included here. Therefore, this set of promising results has encouraged the authors to pursue a detailed analysis of the flow characteristics within the impeller and also at both inlet and discharge sections. Nevertheless, more validations of the numerical results (comparing them with more experimental data) will be presented in forthcoming sections, regarding the flow structure in terms of axial and tangential velocity components, mainly downstream of the turbomachine exit.

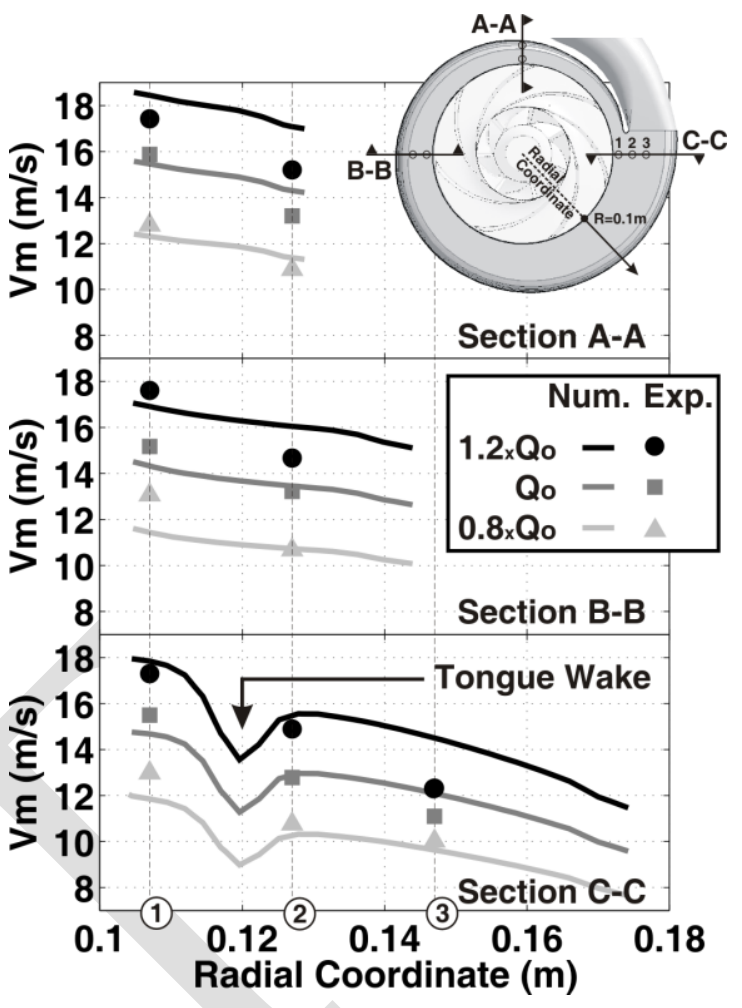

Fig. 7. Numerical-experimental comparative.

Inflow Patterns as a function of the turbine operating conditions. The flow structure at the impeller inlet conditions the performance of the inverse operating mode of the commercial pump. The previous section hinted that the flow is well-distributed by the volute geometry, even though it was designed as a diffuser for the flow discharge. Now, a detailed analysis of the flow patterns entering the impeller will be presented, in order to provide an enhanced picture of the turbine admission. The numerical capabilities will be exploited to describe the flow structure in the relative frame of reference, showing the unsteady features when the pump-turbine operates at the best efficiency point. An assessment of the profile losses will be outlined by studying the inflow angle at the runner inlet, by means of a comparison between its mean value and the geometrical blade angle. Also, the influence of the volute tongue on the circumferential gradients will be described.

Figure 8 illustrates the unsteady evolution of the flow structure at the runner inlet, observed from the moving reference frame. The circumferential section of the runner inlet has been converted into a planar, rectangular domain, representing the impeller width in the ordinates and the impeller perimeter in the abscissa. Also, five intermediate snapshots during a blade passing period $\left(T_{R}\right)$ have been represented to portray the temporal periodicity of the flow unsteadiness. Since the plots are drawn in the relative domain, the observer is watching the displacement of the tongue position, 
superimposed on the fixed blade-to-blade gradients. The upper figure represents the static pressure fields, made non-dimensional by the net head supply at the turbine inlet, while the lower graphic plots the distribution of the unsteady, relative inflow angle (with reference to the impeller blades). In both representations, the blockage induced by the blades is clearly identifiable. As expected, maximum values of non-dimensional static pressure are concentrated towards the leading edges of the blades, revealing the stagnation conditions of the profiles. Also, a noticeable blade-to-blade gradient, present in all the passages, shows the differences between pressure and suction sides and characterizes the blade loadings. Moreover, the discontinuity associated with the tongue position gives an idea of the progressive pressure decay developed in the turbine admission: the impact of the tongue at the inlet is felt as an abrupt reduction of the static pressure, superimposed on the passage structures. Focusing on the distributions of the inflow angle, this contribution of the tongue discontinuity seems not to be as severe as in the case of the pressure fields: an abrupt discontinuity is replaced just by a blockage region with low velocity angles. However, though no overall disturbance is observed, the effect of the tongue is really significant within the blades' passages, because each of the seven passages presents a different blade-to-blade gradient. This is more evident as those passages close to the tongue are considered. On the contrary, it is remarkable that axial gradients are quite negligible in both maps.
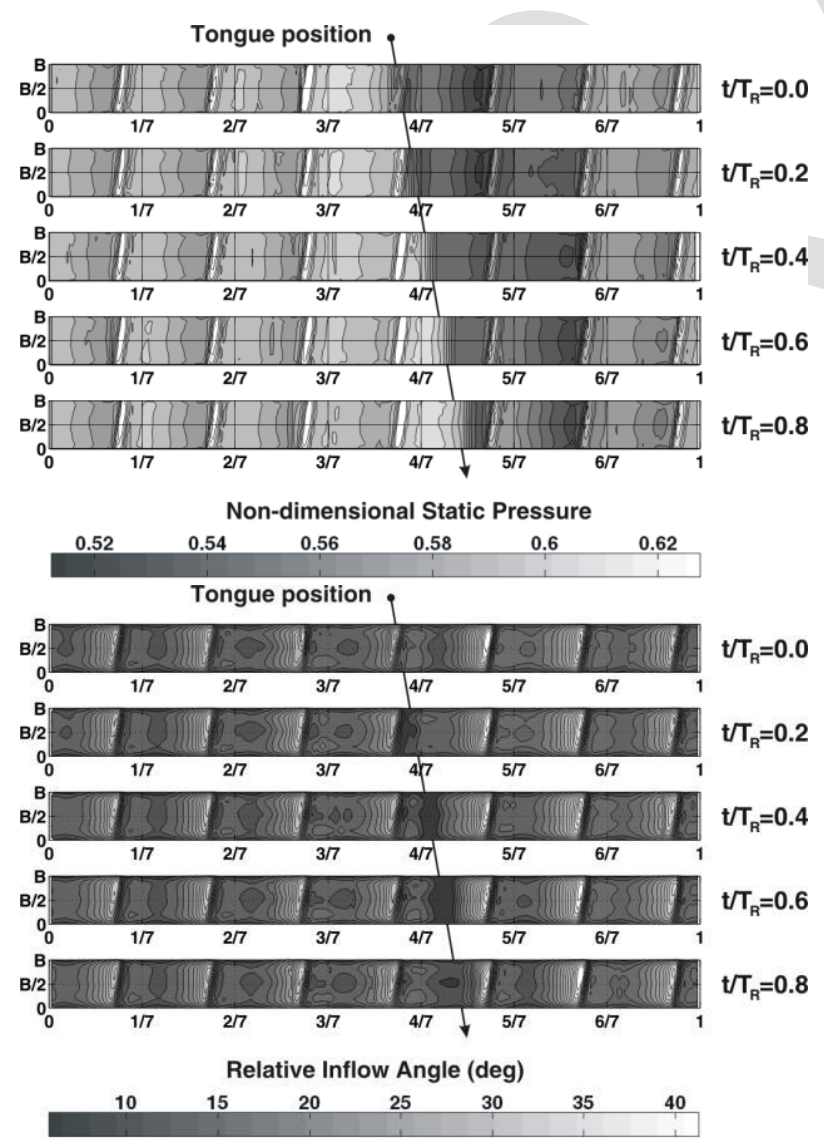

Fig. 8. Unsteady static pressure field and relative flow angle at the impeller inlet (relative frame).
The previous figure 8 is useful in providing a global picture of the flow patterns. However, large variations close to the blade surfaces, for instance in the scale of the flow angle, do not allow a precise comparison between the mean value and geometrical blade angle. To overcome this problem, a one-dimensional diagram of the flow angle has been derived from previous maps, taking into account only the middle line of the impeller width. The time-averaged representation of the flow angle is represented for all the blade passages in figure 9. The averaged contribution of the tongue, shifted along one blade passage, is clearly appreciable. Also, since the temporal averaging is done in the relative frame of reference, the blade-to-blade variations from the suction side to the pressure side of the blades are preserved in the drawings. Finally, the mean value of the angle distribution in every passage is calculated and represented by a dotted line in the figure. Two important conclusions can be drawn from this final result. Firstly, the significant impact of the tongue's non-uniformity, not only influences the closest passages, but also modifies the whole circumferential pattern. And second, the overall mean value of the dotted line being very close to the $29 \mathrm{deg}$ of the blade angle (see table 1), this result resumes the accurate guidance of the inlet flow, in concordance with the global performance of the best efficiency point, and advancing minor profile losses.

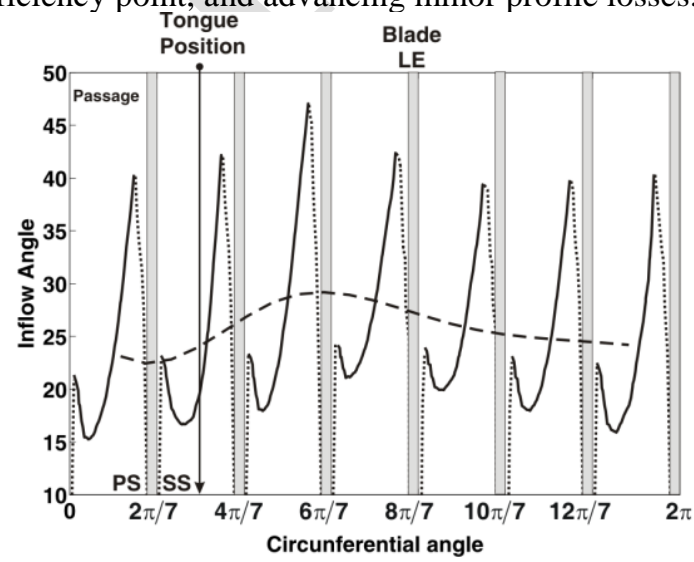

Fig. 9. Averaged impact of the tongue nonuniformity onto the blade-to-blade distributions of the inflow angle.

The analysis of the inflow patterns of the pumpturbine is complemented with the presentation of numerical results for other flow rate conditions in figure 10. In this case, only time-averaged representations have been reproduced. Conversely, the results are shown in the absolute reference frame, so the blade-toblade gradients are smeared out in these maps: only the impact of the non-uniformity derived by the tongue is preserved. With respect to the non-dimensional static pressure, it is observed that the discontinuity associated with the volute tongue is more evident as the flow rate increases, with no axial variations arising in the flow patterns. In addition, the distributions of the inflow prerotation reveal the blockage of the tongue in all cases, which it is quite independent of the flow rate conditions (in both terms of deficit and spatial distortion). 


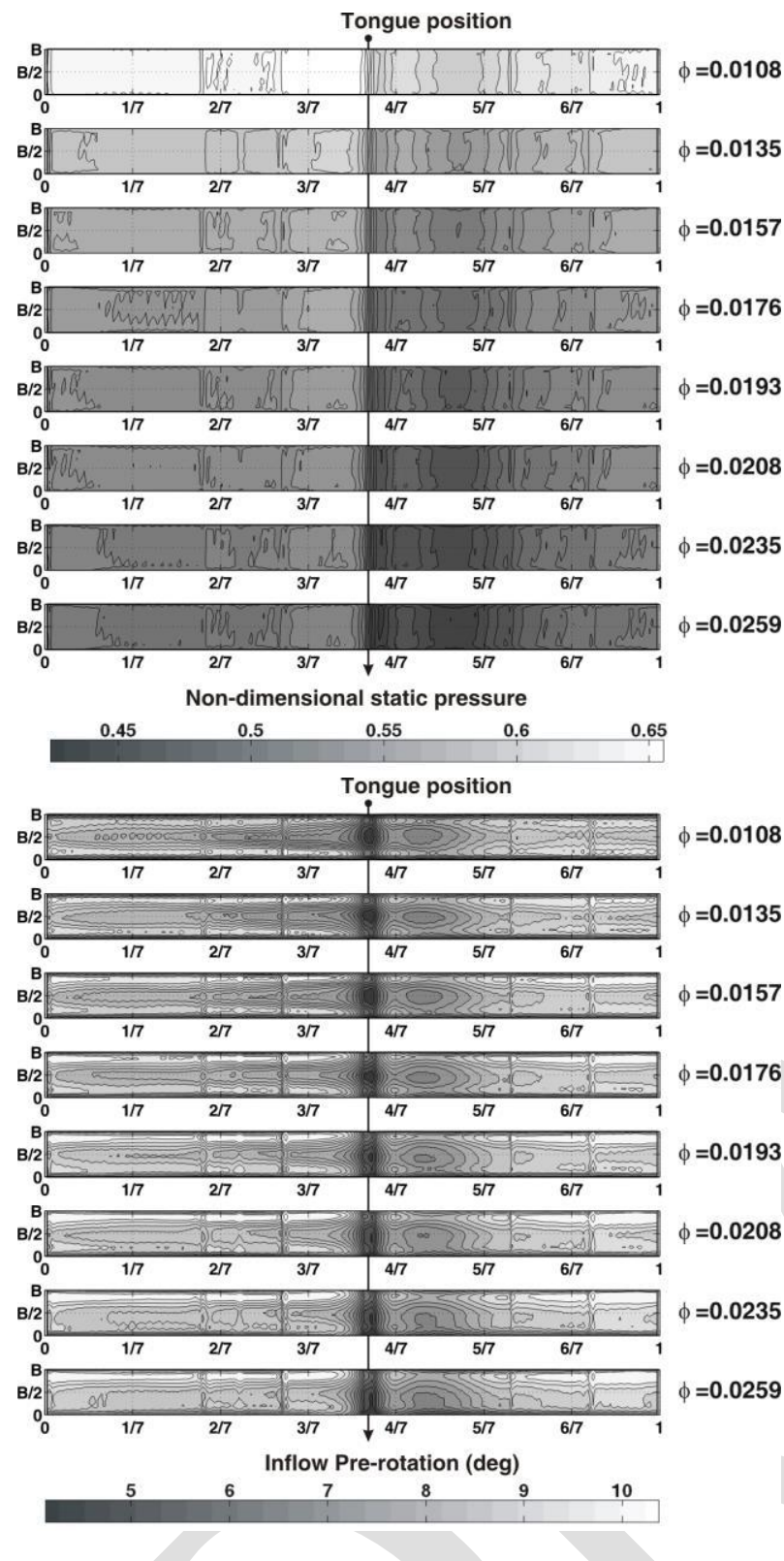

Fig. 10. Time-averaged flow patterns of the static pressure field and the inflow pre-rotation for several flow rate conditions (absolute frame of reference).

A very important feature, related to the profile losses in the impeller blades, is the analysis of the inlet blade load for different flow rate conditions. For that purpose, a diagram similar to figure 9 has been constructed for all the cases executed in the simulation. However, data from all the passages have been reduced (averaged) to a representative one, in order to minimize the influence of the tongue distortion. Therefore, figure 11 represents the passage-averaged distribution of the time-averaged inflow angle as a function of the operating conditions. The line representing the best efficiency point $(\phi=0.0135)$ is centred on the 29-deg reference value of the blade angle, as expected. As the flow rate increases, the differences between pressure and suction sides grow. Also, the mean flow angle diverges from the optimal, thus increasing the profile losses. Furthermore, the flow conditions near the impeller blades are progressively affected. Beneath the pressure side, huge regions with detached conditions develop as a consequence of the progressive underturning of the flow streamlines at higher flow rates. This is clearly observed in the numerical results for moderate and high flow coefficients. A vector map has been included in the top right-hand corner of figure 11 to illustrate the recirculation structure. Complementarily, this deviation in the incidence flow sets off a jet-like impingement over the suction side of the blades, reducing even more the hydraulic performance of the profiles. This phenomenon is mainly observed in the tip regions of the blades, diverting the flow towards the pressure side and reinforcing the separation of the pressure side (see top left-hand corner of the figure). Notice also that the mean inflow angle of the distributions is severely increased for higher flow rates, reaching values between 60 and 85 degrees, completely unacceptable for a stable operation of the pump working as a turbine: vortex shedding coming from detached regions as well as pulsating oscillations of the jet flow impingement over the blade surfaces will arise as major sources of radial imbalance, which may cause important mechanical strains and high tonal noise generation.

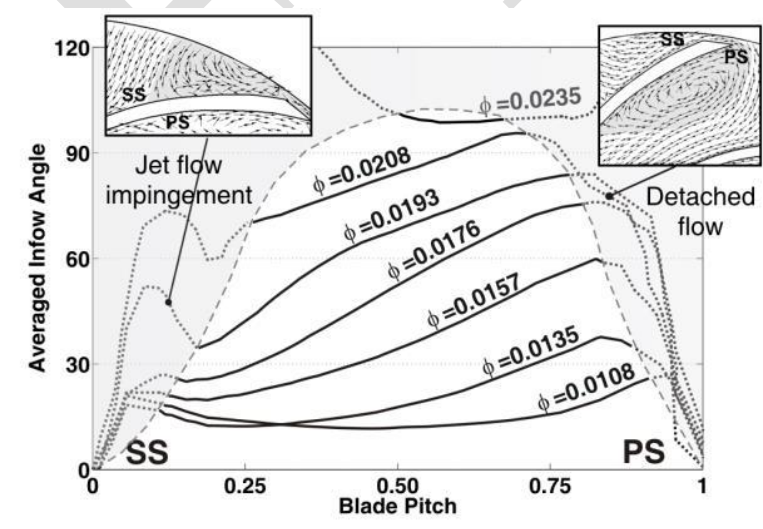

Fig. 11. Blade-to-blade distributions of the averaged inflow angle as a function of the flow rate.

These mechanisms are related to the high unsteady fluctuations of the radial force over the impeller, limiting the practical operation of the commercial pump working as a turbine to only those conditions close to the best efficiency point.

Flow streamlines within the impeller passages. The impact of the operating conditions over the inlet flow is also illustrated in figure 12 , where the streamlines within the impeller passages are represented. Timeaveraged values have been post-processed to calculate the streamlines, so the tongue disturbance is filtered out for these final results. At lower flow rates, there is a clear overturning induced by the deficit of the radial velocity associated with the reduction in the flow rate, as predicted by kinematic considerations. Thus, the relative velocity vectors are excessively rotated, leading to partial detachments in the suction side of the blades $(\phi=0.0061)$. Moreover, due to the poor guidance found in the impeller, a leakage flow is established at 
the tip region of the blades, leading to a severe flow migration from one passage to the adjacent. At nominal conditions, the guidance of the flow is notably improved, so profile losses are minimal: the flow is well-distributed, with no relevant secondary flows affecting the energy exchange. At higher flow rates $(\phi=0.0193,0.0247)$, the limiting mechanisms discussed in figure 11 are again observed in the traces of the streamlines. The higher the flow rate, the more important the impact of the fully-detached regions on the pressure side of the blades is. As the flow is underturned due to the excess of mass flow rate, streamlines are impinging normal to the suction surface. Practically, one half of the blade is not properly working, as deduced from the partial leakage of flow from the suction to the pressure side of the blades. This is the real motor of the development of a recirculation structure beneath the blades.
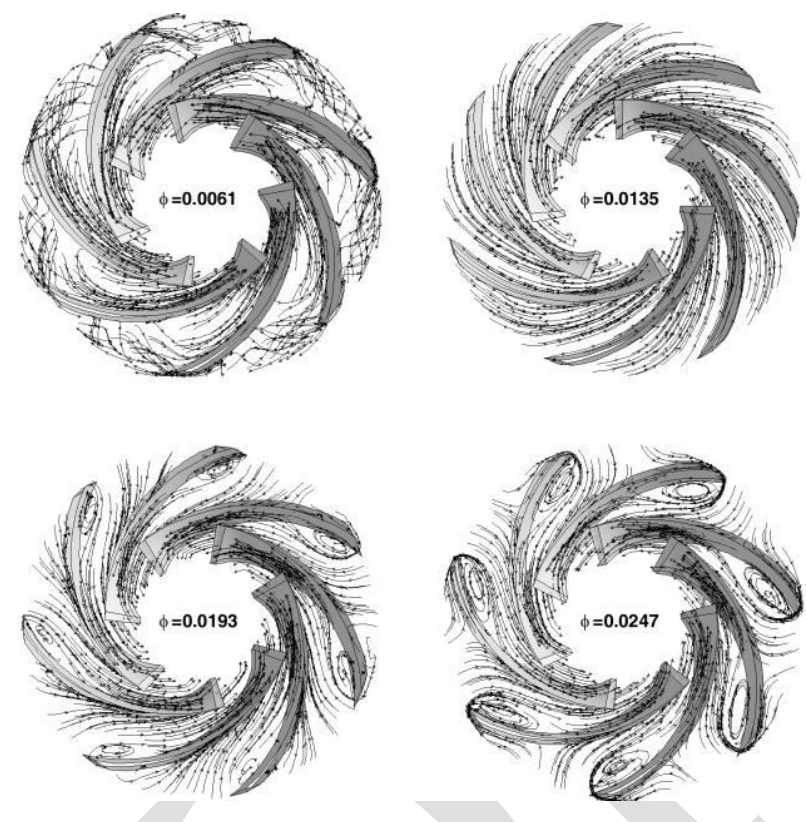

Fig. 12. Effect of the operating conditions on the streamlines through the impeller passages.

Flow structures at the turbine discharge. Intensive measurements were conducted at the turbine discharge to characterize the residual dynamic energy at the turbine outlet. A test section, placed $18 \mathrm{~mm}$ downstream of the impeller (see sketch in figure 13), was selected to conduct several tests using the pneumatic probe designed ad hoc for this investigation. The effect of the operating condition was taken into account as usual, measuring for three different flow rates: best efficiency point and both 80 and $120 \%$ of the nominal conditions. Also, up to ten radial positions were employed in the experiments, so a detailed radial distribution of both axial and tangential velocities could be provided. In addition, two different angular positions (with a 45 degree angular separation between them) were analysed to evaluate the residual effect of the tongue nonuniformity on the outlet flow structures. However, no significant differences were found along the circumferential coordinate, so only the results from one angular position have been reproduced here.

Figure 13 compares the experimental results (represented by points) with those extracted from the numerical model (plotted with lines). Once again, the numerical data are obtained after time-averaging the unsteady solution. The radial distribution of the axial velocity, represented with dotted lines, has been made non-dimensional with the overall mean axial velocity for each mass flow rate. The tangential velocity, in solid lines, is normalized by the tip blade velocity. The distribution of tangential velocity is established as an indicator of the residual energy, non-transferred into net work in the turbine impeller. It will be dissipated into losses downstream. For nominal conditions (dark grey), zero-centred values are found in the outer regions of the section, confirming the good performance at the best efficiency point with an accurate guidance and a real axial flow discharge. Inner zones are influenced by the blockage on the nose impeller, introducing perturbations and secondary flows. Other flow rate conditions present important tangential velocities, reaching even 10 or $15 \%$ of the blade tip velocity. With respect to the distribution of axial velocity, a low-velocity region is also developed, associated with the interference of the impeller nose. Higher flow rates may even experience reverse flow near the impeller hub (radial coordinates of 5 to $10 \mathrm{~mm}$ ), as a consequence of the extremely poor inlet conditions. Notice that the radial profile of axial velocity shows that it is necessary to locate the outlet boundary conditions far beyond the impeller exit, as a good practice in the modelling. Otherwise, high gradients would be neglected and mixing-out mechanisms associated with dissipation of residual energy could not be numerically resolved. Additionally, the figure confirms that overall trends are well-followed by the numerical modelling, in both axial and tangential components. Swirl velocities, derived from the turning angles imposed by the impeller blades, present an excellent agreement. On the contrary, the axial profile is more abrupt for the numerical prediction, indicating an underestimation of the mixing-out process at the turbine exit. It is well-known that precise simulations of mixing mechanisms (jet shear layers, wake decays, detached blockages) require extremely fine meshes to capture most of the flow scales involved in such complex processes. With coarse meshes (mandatory at the domain exit, in order to obtain affordable computation times), momentum transfer is underpredicted, and axial deficits in the wake fluid cannot be modelled with highorder precision. 


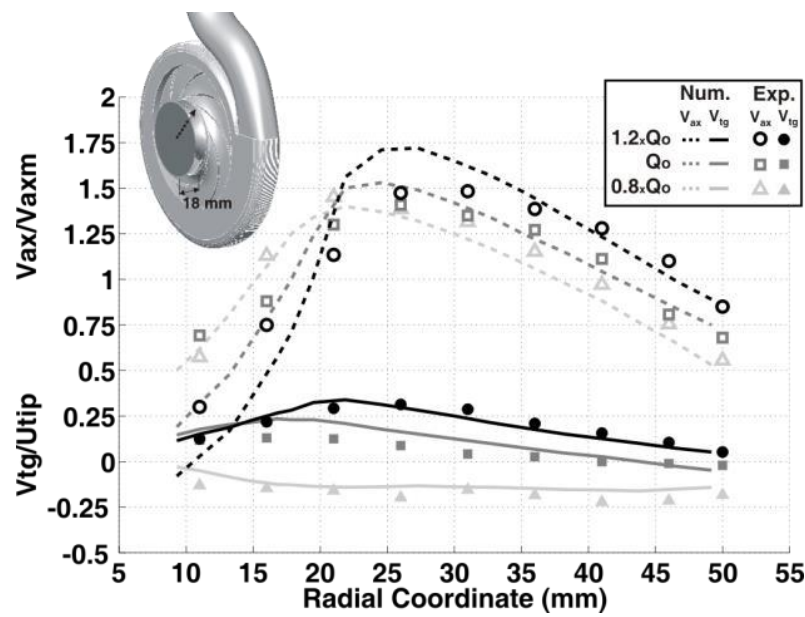

Fig. 13. Numerical-experimental comparative of axial and tangential velocities at the turbine exit.

The impact of the tongue non-uniformity on the outflow patterns is now shown in figure 14. In the experimental dataset, it was previously shown that no significant effects were found in the velocity distributions in a section of the outlet pipe. Now, concerning the numerical cases, strictly the outlet plane of the impeller is analyzed. Here, a slight contribution is observed on the time-averaged results, as indicated in the maps of non-dimensional axial velocity introduced in figure 14. Also, the flow pattern is completely opposite to that observed at the outlet pipe: the flow is clearly concentrated over the impeller hub, with a clear radial gradient of decreasing axial velocity towards the tip region. The radial gradient is dominant,
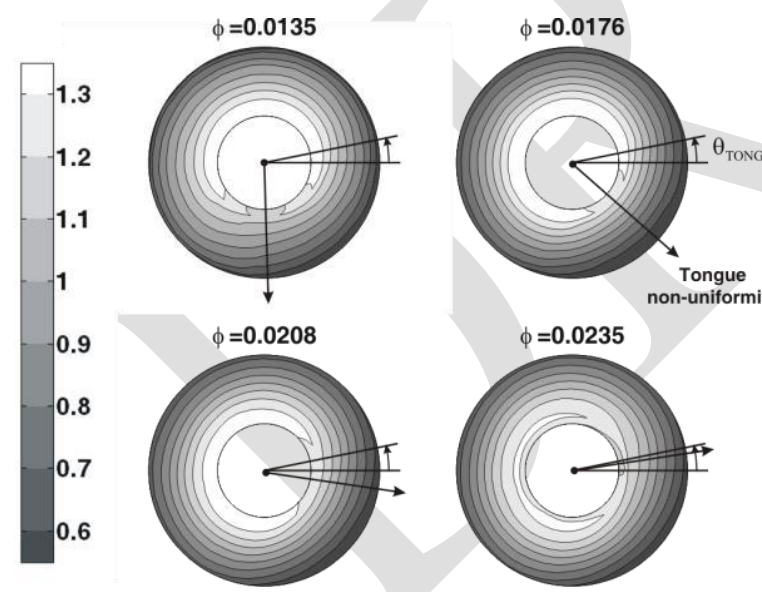

Non-dimensional Axial Velocity

Fig. 14. Distribution of axial velocity at the impeller exit as a function of the mass flow rate.

so the circumferential distortion derived from the tongue non-uniformity is partially masked in the drawings. However, it is remarkable that the nonuniformity is manifested at different circumferential positions of the outlet plane, depending on the flow rate conditions. As the mass flow rate is increased, the delay time for the bulk flow to pass by the impeller is reduced, so the circumferential transport of the disturbance is modified. For all the cases represented, the geometrical position of the volute tongue is indicated in the maps as $\theta_{\text {volute }}$. Also the non-uniformity, which is transported clockwise by the impeller, has been marked with arrows to evaluate the tangential displacement of this flow pattern.

Finally, this section concludes by reporting the spanwise evolution of the relative flow angle at the impeller outlet (figure 15). To obtain this figure, it was necessary to perform a time-averaging of the unsteady computations and then an ensembling procedure to reduce all data to a single blade passage. Thus, a last pitch-averaging allows us to represent a radial distribution (throughflow) of the outflow angle. To compute the angle, the following trigonometric relationship was employed: $\beta_{2}=\operatorname{arctg}\left(v_{a x} /\left(\Omega r+v_{\theta}\right)\right)$.

The results are compared to the spanwise distribution of the blade angles, which ranges from $17 \mathrm{deg}$ at the tip to $32 \mathrm{deg}$ at the hub (plotted with a grey line). At nominal conditions ( $\phi=0.0135$ ), the flow is well-guided by the blades, following the geometrical angle especially in the inner half of the span. Towards the tip, secondary flows arise and the good performance of those sections is lost. The figure also includes the velocity triangles of the flow at midspan.
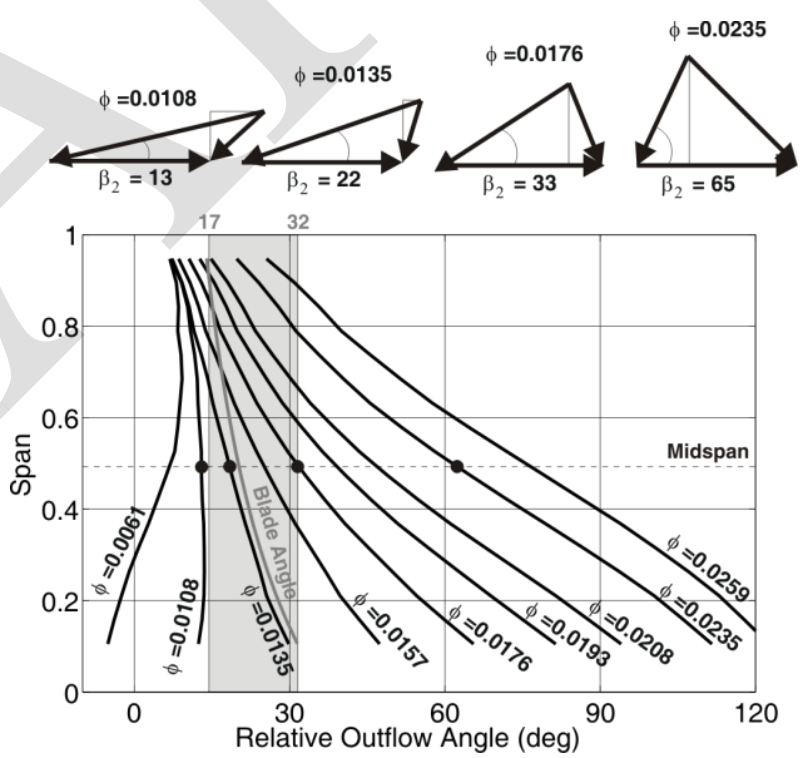

Fig. 15. Radial distributions of outflow angle (throughflow) for different operating conditions.

Note that for the best efficiency point, the absolute velocity is almost axial, as desirable for no mixing losses. For higher flow rates, the exit angle (relative to the blade) is progressively increased, increasing the absolute tangential velocity and contributing to a high level of residual energy at the exit. Excessive mass flow rates cannot be properly managed by the impeller, due to the limited number of blades. Although the cross-area of the passages is restricted at the exit, the large amount of ingested flow is barely guided by the blades. This is clearly pointed out in the figure: the differences established between the turning angles of the blades 
(grey line) and the real distributions followed by the fluid are very large, reaching more than $60 \mathrm{deg}$.

\section{CONCLUSIONS}

A full-3D unsteady numerical simulation of a commercial pump operating as a turbine has been carried out using the sliding mesh technique with accurate time stepping. A previous experimental dataset has been employed to validate the global performance predicted by the code, as well as to check local numerical results at both inlet and outlet sections of the turbomachine. In this investigation, the numerical model has been used to explore the operational characteristics of pump-designed impellers when working in the inverse mode, especially concerning the unsteady forces and the flow patterns throughout the blade passages.

Basically, the study demonstrates that the commercial design of the pump allows a reasonable use of the impeller as a turbine runner. In particular, there is a significant range of mass flow rates (centred on the best efficiency point of the inverse mode) where there is an accurate guidance of the flow in both inlet and outlet sections of the impeller. Moreover, the volute casing has demonstrated its ability to provide a uniform distribution of the flow at the turbine admission, assuring minimum profile losses for the best efficiency point. Moreover, the efficiency of the pump working as a turbine is almost as high as that achieved for the direct operational mode.

Regarding the mechanical stresses on the impeller, it has been shown that both axial and radial forces are controlled, exhibiting values very similar to those registered in the pumping mode. Special care must be taken with the unsteady oscillations of these forces, mainly when working beyond the nominal intervals. In that case, high levels of unsteadiness clocked with the blade passing frequency may produce severe dynamic loads. These fluctuations, derived from a strong bladetongue interaction, can be as high as $25 \%$ of the steady component, constituting a serious risk of fatigue failure of the mechanical components. Nevertheless, a moderate use of the pump as a turbine, limited to a few hours and mainly working at nominal conditions, will meet perfectly all the requirements of small hydraulic resources.

Additionally, an extensive analysis of the numerical results has provided insight into the unsteady flow patterns inside the impeller. Both absolute and relative reference frames have been considered in order to present the impact of the tongue disturbances on the circumferential blade-to-blade gradients within the impeller passages. The influence of the operating conditions on the primary flow patterns has also been addressed, providing valuable information to explain the characteristics of the flow structure developed in a commercial pump working as a turbine.

\section{ACKNOWLEDGEMENT}

The research conducted has been sponsored by the "Ministerio de Ciencia e Innovación" (Spain) under
Projects No. DPI2006-15720, DPI2006-15638-C02-02 and TRA2007-62708.

\section{NOMENCLATURE}

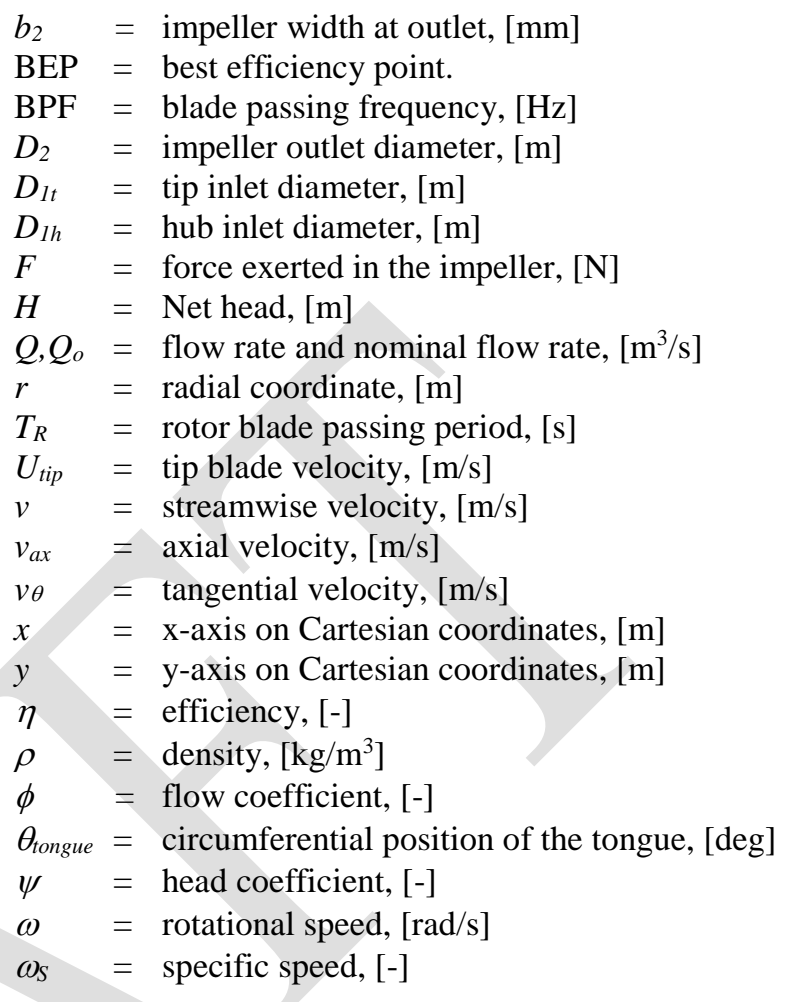

\section{REFERENCES}

1. Kittredge, C.P. Centrifugal pumps used as hydraulic turbines. Transactions of the ASME Journal of Engineering for Power 1961; 83:74-78.

2. Knapp, R.T. Centrifugal Pump Performance as Affected by Design Features. Transactions of the ASME, 1941; 63:241250.

3. Stepanoff, A. J. Centrifugal and Axial Flow Pumps. John Wiley \& Sons, New York, 1957.

4. Shafer, L.L. Pumps as Power Turbines. Mechanical Engineering, 1982.

5. Engeda, A., Rautenberg, M. Performance of centrifugal pumps as hydraulic turbines. Transactions of the 4th ASME International Hydro \& Power Fluid Machinery Symposium, Anaheim CA 1987; 43.

6. Priesnitz, C. Einsatzmöglichkleiten von rückwärtslaufenden Standardkreiselpumpen als Turbinen zur Energierückgewinnung. Pumpen und Verdichter Informationen, 1987, 3-12.

7. Shafer L., Agostinelli A. Hydraulic Pump in Reverse Makes Good Low Cost Hydroturbine, Power, 1981.

8. Thoma, D., Kittredge, C.P. Centrifugal Pumps Operated under Abnormal Conditions. Power 1931, 881-884.

9.Swanson, M.W. Complete characteristics circle diagram for turbomachinery. Transactions of the ASME 1953; 75:816826 Vol. 75, 1953.

10. Patterson I.S., Martin C.S. Effect of specific speed on pump characteristics and hydraulic transients in abnormal zones of operation, Proceedings of the 12th IAHR Symposium, Stirling 1984, 151-172.

11. Williams A.A. The turbine performance of centrifugal pumps: a comparison of prediction method. Proceedings of 
the Institution of Mechanical Engineers. Part A. Journal of power and energy 1994; 208:59-66.

12. Derakhshan, S., Nourbakhsh, A. Experimental study of characteristic curves of centrifugal pumps working as turbines in different specific speeds. Experimental Thermal and Fluid Science 2008; 32:800-807.

13. Tamm, A., Braten, A., Stoffel, B. And Ludwig, G. Analysis of a Standard Pump in Reverse Operation Using CFD. Proceedings of the 20th IAHR Symposium, Charlotte, NC (USA), 2000

14. González J., Fernández Oro, J.M., Argüelles Díaz, K.M., Santolaria Morros C. Flow Analysis for a Double Section Centrifugal Machina in the Pump and Turbine Operation Modes, International Journal for Numerical Methods in Fluids, in-press (on-line), 2008.

15. Fernández J., Santolaria C., Ballesteros R., Blanco E. Test facility of inverse-working pumps for small hydro-power plants. Proceedings of International Congress on Hydropower into de Next Century, Barcelona (Spain) 1995, 657-664.

16. González, J., Santolaria, C. Unsteady Flow Structures and Global Variables in a Centrifugal Pump, ASME Journal of Fluids Engineering 2006; 128: 937-946.

17. Freitas C.J. Journal of Fluids Engineering Editorial Policy Statement on the Control of Numerical Accuracy. ASME Journal of Fluids Engineering 1993; 115:339-340.
18. Brennen, C.E. Hydrodynamics of Pumps. Oxford University Press and CETI Inc, Oxford. 1994.

19. González, J.; Parrondo, J.; Santolaria, C, Blanco, E. Steady and Unsteady Radial Forces for a Centrifugal Pump with Impeller to Tongue Gap Variation. ASME Journal of Fluids Engineering 2006; 128:454-462.

20. Neumann, B. The Interaction between Geometry and Performance of a Centrifugal Pump, Mechanical Engineering Pub. Ltd., London, 1991.

21. Croba D., Kueny, J.L. Numerical calculation of 2D, unsteady flow in centrifugal pumps: impeller and volute interaction. International Journal for Numerical Methods in Fluids 1996; 22:467-481.

22. Shi, F., Tsukamoto, H. Numerical study of pressure fluctuations caused by impeller-diffuser interaction in a diffuser pump stage. ASME Journal of Fluids Engineering 2001; 123:466-474.

23. González, J.; Fernández, J.; Blanco, E.; Santolaria, C.; Numerical Simulation of the dynamic effects due to impeller-volute interaction in a centrifugal pump. ASME Journal of Fluids Engineering, 2002; 124:348-355. 
This document is a pre-print version of the scientific paper published by Wiley. It has been released by the authors to fulfill all the publisher requirements established for Article Sharing:

https://authorservices. wiley.com/author-resources/Journal-

Authors/licensing/self-archiving.html

This is the pre-peer reviewed version of the article which has been published in final form at the International Journal of Numerical Methods in Fluids. This version may be used for non-commercial purposes in accordance with Wiley

Terms and Conditions for Use of Self-Archived Versions

\section{(2) $\odot \Theta \Theta$}

(C) 2019. This manuscript version is made available under the Creative Commons Attribution-NonCommercial-NoDerivatives 4.0 International License (CC-BY-NC-ND 4.0 license) http://creativecommons.org/licenses/by-nc-nd/4.0/ 